\title{
Simultaneous Measurability of Error and Disturbance
}

\author{
Riuji Mochizuki \\ Laboratory of Physics, Tokyo Dental College, Tokyo, Japan \\ Email: rjmochi@tdc.ac.jp
}

Received November 19, 2012; revised December 21, 2012; accepted December 30, 2012

\begin{abstract}
The uncertainty relation, which displays an elementary property of quantum theory, was originally described by Heisenberg as the relation between error and disturbance. Ozawa presented a more rigorous expression of the uncertainty relation, which was later verified experimentally. Nevertheless, the operators corresponding to error and disturbance should be measurable in the identical state if we follow the presupposition of Heisenberg's thought experiment. In this letter, we discuss simultaneous measurability of error and disturbance and present a new inequality using error and disturbance in the identical state. A testable example of this inequality is also suggested.
\end{abstract}

Keywords: Quantum Logic; Uncertainty; Inequality

\section{Introduction}

The uncertainty relation, which displays an elementary property of quantum theory, was originally described by Heisenberg [1] as the relation between the error $\epsilon$ and disturbance $\eta$ of a particle's position and momentum as

$$
\epsilon \eta \geq h,
$$

where $h$ is Planck's constant.

Subsequently, a more generalized inequality was shown $[2,3]$ :

$$
\sigma(A) \sigma(B) \geq 12|\langle[A, B]\rangle|,
$$

where $\sigma(X)$ is the standard deviation of a self-conjugate operator $X$, which corresponds to some physical quantity, defined as

$$
\sigma(X)=\left\langle(\Delta X)^{2}\right\rangle^{1 / 2}
$$

with

$$
\Delta X=X^{\text {in }}-\left\langle X^{\text {in }}\right\rangle,
$$

and $[A, B]$ as the commutator of $A$ and $B$.

In some literature (for example, [4]), (2) is considered to be a more formal expression of (1).

Several decades later, Ozawa presented a more rigorous expression of the uncertainty relation [5-7]. The rootmean-square noise $\epsilon(A)$ and root-mean-square disturbance $\eta(B)$ are defined as

$$
\begin{aligned}
& \epsilon(A)=\left\langle N(A)^{2}\right\rangle^{1 / 2}, \\
& \eta(B)=\left\langle D(B)^{2}\right\rangle^{1 / 2} .
\end{aligned}
$$

The Noise operator $N(A)$ is defined using the meter-observable $M^{\text {out }}$ of $A^{\text {in }}$ as

$$
N(A)=M^{\text {out }}-A^{\text {in }},
$$

with the disturbance operator $D(B)$ as

$$
D(B)=B^{\text {out }}-B^{\text {in }},
$$

where in and out mean just before and just after measurement, respectively. The new uncertainty relation is written by means of (5), (6) and also (3) as

$$
\begin{aligned}
& \epsilon(A) \eta(B)+\epsilon(A) \sigma(B)+\sigma(A) \eta(B) \\
& \geq 1 / 2\left|\left\langle\left[A^{\text {in }}, B^{\text {in }}\right]\right\rangle\right|
\end{aligned}
$$

Recently, it was reported [8] that (9) was verified experimentally by a neutron spin experiment. Nevertheless, it is not clear whether verification of (9) is possible for continuous quantities such as position and momentum. In other words, it is not clear whether (5) and (6) are measurable for such quantities [9,10]. Watanabe et al. [11-13] suggested another inequality suitable for practical measurement.

Moreover, error and disturbance were defined in the identical state in Heisenberg's thought experiment [1] referring to the uncertainty principle. If we follow his presupposition, the operators corresponding to error and disturbance should be simultaneously measurable. In many textbooks on quantum theory, commutativity of observables is regarded as a necessary and sufficient condition of possibility of simultaneous measurement. Ozawa, however, insists in his paper [14] that, in some states, two noncommutative observables, $A$ and $B$, are 
simultaneously measurable if they satisfy

$$
\epsilon(A)=\epsilon(B)=0
$$

and their meter observables are commutative. Simultaneous measurability has been discussed with respect to contextuality and weak measurement [14-17].

The purpose of this letter is to discuss the simultaneous measurability of error and disturbance. Firstly, we define simultaneous measurability from the quantum logical aspect. According to our definition, there exists no state where noncommutative observables are simultaneously measurable. Then, we define commutative operators which correspond to the error and disturbance of noncommutative observables. This definition leads to the uncertainty relation of error and disturbance in the identical state. A testable example of this relation is also suggested, where definition of error $\epsilon$ in [8] is shown to be insufficient for other settings.

\section{Simultaneous Measurability}

To prepare for discussion about simultaneous measurability, we define observables according to a common quantum logical approach $[18,19]$. The proposition that a measured value of a physical quantity $u$ belongs to a subspace $A$ of space of real number $R$ is written as $u(A)$. When the truth value of $u(A)$ can be determined experimentally, $u$ is called measurable. Logic $L$, which is nothing but a $\sigma$-complete orthomodular lattice, consists of such propositions. Classical logic is a Boolean lattice, namely, an orthocomplemented distributive lattice, while quantum logic is not.

We suppose $\sigma$-field $\mathcal{B}(\boldsymbol{R})$, which consists of all open sets belonging to space of real number $\boldsymbol{R}$. A map $u$ from $\mathcal{B}(\boldsymbol{R})$ to logic $L$ is called an observable of $L$ if

$$
\begin{gathered}
u(R)=1, u(\varnothing)=0, \\
u(A)^{\perp}=u(\boldsymbol{R}-A) \text { for } A \in \mathcal{B}(\boldsymbol{R}), \\
u\left(\bigcup_{n=1}^{\infty} A_{n}\right)=\bigvee_{n=1}^{\infty} u\left(A_{n}\right) \text { for } A_{n} \in \mathcal{B}(\boldsymbol{R}), \\
\text { if } A_{m} \cap A_{n}=\varnothing \text { for } m \neq n,
\end{gathered}
$$

where $u(A)^{\perp}$ is the orthocomplement of $u(A)$ and $\left\{u\left(A_{n}\right) ; n=1,2, \cdots\right\}$ constitute an orthogonal set of projection operators. It is proved that observables are $\sigma$ homomorphism from $\mathcal{B}(\boldsymbol{R})$ to $L$.

There exists a one-to-one correspondence between the whole set of bounded observables and the whole set of bounded self-conjugate linear operators. If, and only if, two such operators, which correspond to observables $u$ and $v$, are commutative, they satisfy for any pair of $A, B \in \mathcal{B}(\boldsymbol{R})$

$$
v(B)=(v(B) \wedge u(A)) \vee\left(v(B) \wedge(u(A))^{\perp}\right)
$$

and the orthomodular lattice whose elements are $u(A)$ 's and $v(B)$ 's is Boolean. Here, we assume, as usual, that all the measurable quantities are observables.

We define the simultaneous measurability of observables $u$ and $v$ as follows.

\section{Definition}

$u$ and $v$ are called simultaneously measurable if the truth value of $u(A) \wedge v(B)$ can be determined experimentally.

We present the following theorem:

Theorem

Let $u$ and $v$ be observables of logic $L$ and $u_{(v=B)}\left(A_{n}\right) \equiv u\left(A_{n}\right) \wedge v(B) \in L, A_{n}, B \in \mathcal{B}(\boldsymbol{R}), n=1,2, \cdots$ for the fixed $v(B)$. Then, $u_{(v=B)}\left(A_{n}\right), n=1,2, \cdots$ are observables if, and only if, they satisfy (14).

\section{Proof (sufficiency)}

We assume (14) is satisfied. Firstly, we show the whole set $L_{v=B}$ whose elements are $u_{(v=B)}\left(A_{n}\right)$, $n=1,2, \cdots$ is a $\sigma$-complete orthocomplemented distributive lattice. Since $u\left(A_{n}\right)$ 's and $v(B)$ satisfy the distribution law,

$$
\bigvee_{n} u_{(v=B)}\left(A_{n}\right)=\left(\bigvee_{n} u\left(A_{n}\right)\right) \wedge v(B) \in L_{v=B}
$$

and $u_{(v=B)}\left(A_{n}\right)$ also satisfy the distribution law. Moreover, if we define

$$
(u(A) \wedge v(B))^{\perp} \equiv(u(A))^{\perp} \wedge v(B)
$$

for $u(A) \wedge v(B) \in L_{v=B},(u(A) \wedge v(B))^{\perp}$ is the orthocomplement of $u(A) \wedge v(B)$. Thus $L_{v=B}$ is a $\sigma$-complete orthocomplemented distributive lattice. It is clear that $u_{(v=B)}\left(A_{n}\right), \quad n=1,2, \cdots$ satisfy (11)-(13) because $L_{v=B}$ is a distributive lattice. Therefore $u_{(v=B)}\left(A_{n}\right), \quad n=1,2, \cdots$ are observables of $L_{v=b}$ if they satisfy (14). (necessity).

Let $u_{(v=B)}\left(A_{n}\right), n=1,2, \cdots$ be observables. From (13)

$$
u_{(v=B)}\left(A_{m}\right) \vee u_{(v=B)}\left(A_{n}\right)=u_{(v=B)}\left(A_{m} \cup A_{n}\right),
$$

if $A_{m} \cap A_{n}=\varnothing$. This equation leads to

$$
\begin{aligned}
& \left(v(B) \wedge u\left(A_{m}\right)\right) \vee\left(v(B) \wedge u\left(A_{n}\right)\right) \\
& =v(B) \wedge u\left(A_{m} \cup A_{n}\right) \\
& =v(B) \wedge\left(u\left(A_{m}\right) \vee u\left(A_{n}\right)\right) .
\end{aligned}
$$

If we put $A_{n}=R-A_{m}$,

$$
\begin{aligned}
& \left(v(B) \wedge u\left(A_{m}\right)\right) \vee\left(v(B) \wedge u\left(\boldsymbol{R}-A_{m}\right)\right) \\
& =v(B) \wedge\left(u\left(A_{m}\right) \vee u\left(A_{m}\right)^{\perp}\right)=v(B) .
\end{aligned}
$$

QED.

From the above, it is shown that $u(A) \wedge v(B)$ is not an observable if (14) is not satisfied, that is, two observ- 
ables which correspond to mutually-noncommutative linear operators are not simultaneously measurable.

For example, let

$$
\begin{aligned}
& P_{x+}=\frac{1+\sigma_{x}}{2}, \\
& P_{\phi+}=\frac{1+\sigma_{\phi}}{2},
\end{aligned}
$$

be projection operators corresponding to $u(A)$ and $v(B)$, respectively, where

$$
\sigma_{\phi}=\sigma_{x} \cos \phi+\sigma_{y} \sin \phi .
$$

$\sigma_{x}$ and $\sigma_{y}$ are Pauli spin matrices. Then, if $\phi \neq 0$, the projection operator corresponding to $u(A) \wedge v(B)$ is 0 , which is not an observable.

\section{Uncertainty Relation}

From the previous section, we can say such quantities as

$$
\langle N(A) D(B)\rangle
$$

are not measurable because (7) and (8) are noncommutative when $[A, B] \neq 0$. Note that this fact does not deny (9) where (16) does not appear but (5), (6) and (3) do. These are measured separately by using states belonging to the same statistical ensemble. What we would like to emphasize is that the uncertainty relation should be written by means of commutative quantities if it is thought to be the relation between quantities which are measured in the identical state. Thus we define

$$
\begin{gathered}
\mathcal{N}(A)=M^{\text {out }}-\left\langle A^{\text {in }}\right\rangle, \\
\mathcal{D}(B)=B^{\text {out }}-\left\langle B^{\text {in }}\right\rangle,
\end{gathered}
$$

as operators which express error and disturbance from the expectation values, respectively.

Using these operators, we examine the following quantity:

$$
\left\langle\mathcal{N}(A)^{2} \mathcal{D}(B)^{2}\right\rangle^{1 / 2}
$$

Since $M^{\text {out }}$ and $B^{\text {out }}$ are observables in different systems, (19) becomes

$$
\left\langle\mathcal{N}(A)^{2} \mathcal{D}(B)^{2}\right\rangle^{1 / 2}=\left\langle\mathcal{N}(A)^{2}\right\rangle^{1 / 2}\left\langle\mathcal{D}(B)^{2}\right\rangle^{1 / 2}
$$

If we use

$$
\begin{aligned}
& \left\langle\mathcal{N}(A)^{2}\right\rangle^{1 / 2}=\left\langle(N(A)+\Delta A)^{2}\right\rangle^{1 / 2}, \\
& \left\langle\mathcal{D}(B)^{2}\right\rangle^{1 / 2}=\left\langle(D(B)+\Delta B)^{2}\right\rangle^{1 / 2} .
\end{aligned}
$$

and assume

$$
\langle N(A) \Delta A\rangle=\langle D(B) \Delta B\rangle=0,
$$

(19) is written by the use of (3), (5) and (6) as

$$
\begin{aligned}
& \left\langle\mathcal{N}(A)^{2} \mathcal{D}(B)^{2}\right\rangle^{1 / 2} \\
& =\left(\epsilon(A)^{2}+\sigma(A)^{2}\right)^{1 / 2}\left(\eta(B)^{2}+\sigma(B)^{2}\right)^{1 / 2} .
\end{aligned}
$$

It is clear that (22) is not invariably realized. One of the simplest counter examples is the case where $M^{\text {out }}$ always indicates $\left\langle A^{\text {in }}\right\rangle$. Nevertheless, we regard (22) as a rather reasonable assumption, which means that $N(A)$ and $\Delta A$ are independent stochastic variables, and so are $D(B)$ and $\Delta B$.

We can calculate the lower bound of (23) by means of (2) and (9) to obtain

$$
\left\langle\mathcal{N}(A)^{2} \mathcal{D}(B)^{2}\right\rangle^{1 / 2} \geq(2-\sqrt{2})|\langle[A, B]\rangle| .
$$

If we use

$$
\epsilon(A) \eta(B) \geq \frac{1}{2}|\langle[A, B]\rangle|
$$

in place of (9), the minimal value becomes almost double:

$$
\left\langle\mathcal{N}(A)^{2} \mathcal{D}(B)^{2}\right\rangle^{1 / 2} \geq|\langle[A, B]\rangle|
$$

\section{A Testable Example}

In this section, we suggest an experiment with a setting which is a little modified from the experiment in [8] as a testable example of the inequality (24). We define $A, B$ and $O_{A}$ instead of their definition in [8] as

$$
\begin{gathered}
A=O_{A}=\sigma_{x} \sin \theta+\sigma_{z} \cos \theta, \\
B=\frac{\sigma_{y}}{\sqrt{2}}+\frac{\sigma_{z}}{\sqrt{2}},
\end{gathered}
$$

where

$$
0 \leq \theta \leq \frac{\pi}{2}
$$

and $\psi\rangle=|+z\rangle$. (22), which is necessary to conclude with (24), is satisfied in this setting. If the root-meansquare noise $\epsilon(A)$ is completely calculable by using $A$, $B$ and $O_{A}$ as insisted in [8],

$$
\begin{gathered}
\sigma(A)=\sin \theta, \\
\sigma(B)=\frac{1}{\sqrt{2}}, \\
\epsilon(A)=0, \\
\eta(B)=\sin \theta .
\end{gathered}
$$

Then,

$$
\epsilon(A) \eta(B)+\epsilon(A) \sigma(B)+\sigma(A) \eta(B)=\sin ^{2} \theta
$$


and

$$
\left|\left\langle\left[A^{\text {in }}, B^{\text {in }}\right]\right\rangle\right|=\sqrt{2} \sin \theta .
$$

It comes down to that Ozawa's inequality (9) is not realized within $\sin \theta<1 / \sqrt{2}$. This fact seems to show that $\epsilon(A)$ includes uncontrollable error.

Accordingly, we will estimate the range of $\epsilon(A)$, including uncontrollable error, on the assumption that (25) or (9) is realized. We redefine $\epsilon(A)$ as

$$
\epsilon(A)=\left\langle\left(M^{\text {out }}+\delta M-A^{\text {in }}\right)\right\rangle^{1 / 2},
$$

where $\delta M$ is the operator which gives uncontrollable error and is assumed to satisfy

$$
\left\langle\delta M\left(A^{\text {in }}-\left\langle A^{\text {in }}\right\rangle\right)\right\rangle=0 .
$$

This assumption may demand that the angular momentum of the particle should be measured continuously. Then, inequalities corresponding to (26) and (24) will be derived from (23).

Firstly, if we assume (25), $\epsilon(A) \geq \frac{1}{\sqrt{2}}$ independently of $\theta$. Then,

$$
\left\langle\mathcal{N}(A)^{2} \mathcal{D}(B)^{2}\right\rangle^{1 / 2} \geq\left(\frac{1}{2 \sqrt{2} \sin \theta}+\frac{\sin \theta}{\sqrt{2}}\right)|\langle[A, B]\rangle|
$$

The minimum value of the coefficient of the righthand side is 1 when $\sin \theta=1 / \sqrt{2}$.

Next, if (9) is assumed,

$$
\begin{aligned}
& \epsilon(A) \geq 0,\left(\sin \theta \geq \frac{1}{\sqrt{2}}\right) . \\
& \epsilon(A) \geq \frac{\sin \theta(1-\sqrt{2} \sin \theta)}{1+\sqrt{2} \sin \theta},\left(\sin \theta \leq \frac{1}{\sqrt{2}}\right) .
\end{aligned}
$$

Then

$$
\begin{gathered}
\left\langle\mathcal{N}(A)^{2} \mathcal{D}(B)^{2}\right\rangle^{1 / 2} \geq \frac{1}{\sqrt{2}}\left(\sin ^{2} \theta+\frac{1}{2}\right)^{1 / 2}|\langle[A, B]\rangle|, \\
\left(\sin \theta \geq \frac{1}{\sqrt{2}}\right) . \\
\left\langle\mathcal{N}(A)^{2} \mathcal{D}(B)^{2}\right\rangle^{1 / 2} \geq\left(\frac{1+2 \sin ^{2} \theta}{\sqrt{2}+2 \sin \theta}\right)|\langle[A, B]\rangle|, \\
\left(\sin \theta \leq \frac{1}{\sqrt{2}}\right) .
\end{gathered}
$$

The minimum value of the coefficient of the righthand side is $2-\sqrt{2}$ when $\sin \theta=1-1 / \sqrt{2}$.

If

$$
\frac{\left\langle\mathcal{N}(A)^{2} \mathcal{D}(B)^{2}\right\rangle^{1 / 2}}{|\langle[A, B]\rangle|} \leq 1
$$

at some angles and

$$
2-\sqrt{2} \leq \frac{\left\langle\mathcal{N}(A)^{2} \mathcal{D}(B)^{2}\right\rangle^{1 / 2}}{|\langle[A, B]\rangle|}
$$

at each angle are shown experimentally, we can conclude that Inequality (24) is realized. This is also an experimental proof that Ozawa's inequality is correct.

\section{Conclusion}

To summarize, we have defined simultaneous measurability from the quantum logical aspect and conclude that operators corresponding to the error and disturbance should be commutative if they operate in the identical state. Moreover, a new inequality using such operators and a testable example are presented.

\section{REFERENCES}

[1] W. Heisenberg, "Über den Anschaulichen Inhalt der Quantentheoretischen Kinematik und Mechanik," Zeitschrift für Physik, Vol. 43, No. 3-4, 1927, pp. 172-198. doi:10.1007/BF01397280

[2] E. H. Kennard, "Zur Quantenmechanik Einfacher Bewegungstypen," Zeitschrift für Physik, Vol. 44, No. 4-5, 1927, pp. 326-352. doi:10.1007/BF01391200

[3] H. P. Robertson, "The Uncertainty Principle," Physical Review, Vol. 34, No. 1, 1929, pp. 163-164. doi:10.1103/PhysRev.34.163

[4] J. von Neumann, "Die Mathematische Grundlagen der Quantenmechanik," Springer Verlag, Berlin, 1932.

[5] M. Ozawa, "Universally Valid Reformulation of the Heisenberg Uncertainty Principle on Noise and Disturbance in Measurement," Physical Review, Vol. A67, No. 4, 2003, 6 p. doi:10.1103/PhysRevA.67.042105

[6] M. Ozawa, "Physical Content of Heisenberg's Uncertainty Relation: Limitation and Reformulation," Physics Letters A, Vol. 318, No. 1-2, 2003, pp. 21-29. doi:10.1016/j.physleta.2003.07.025

[7] M. Ozawa, "Uncertainty Relations for Noise and Disturbance in Generalized Quantum Measurements," Annals of Physics, Vol. 311, No. 2, 2004, pp. 350-416. doi:10.1016/j.aop.2003.12.012

[8] J. Erhart, S. Sponar, G. Sulyok, G. Badurek, M. Ozawa and Y. Hasegawa, "Experimental Demonstration of a Universally Valid Error-Disturbance Uncertainty Relation in Spin-Measurements," Nature Physics, Vol. 8, 2012, 11 p. doi:10.1038/nphys 2194

[9] R. F. Werner, "The Uncertainty Relation for Joint Measurement of Position and Momentum," Quantum Infformation and Computation, Vol. 4, 2004, Article ID: arXiv:quant$\mathrm{ph} / 0405184$.

[10] K. Koshino and A. Shimizu, "Quantum Zeno Effect by General Measurements," Physics Report, Vol. 412, No. 191, 2005, Article ID: arXiv:puant-ph/0411145.

[11] Y. Watanabe, T. Sagawa and M. Ueda, "Optimal Meas- 
urement on Noisy Quantum Systems," Physical Review Letters, Vol. 104, No. 2, 2010, 4 p.

doi:10.1103/PhysRevLett.104.020401

[12] Y. Watanabe, T. Sagawa and M. Ueda, "Uncertainty Relation Revisited from Quantum Estimation Theory," Quantum Physics, Article ID: arXiv:1010.3571[quant-ph].

[13] Y. Watanabe and M. Ueda, "Quantum Estimation Theory of Error and Disturbance in Quantum Measurement," Article ID: arXiv: 1106.2526[quant-ph]

[14] M. Ozawa, "Quantum Reality and Measurement: A Quantum Logical Approach," Foundation of Physics, Vol. 41, 2011, pp. 592-607.

[15] J. Dressel, S. Agarwal and A. N. Jordan, "Contextual
Values of Observables in Quantum Measurements," Physical Review Letters, Vol. 104, 2010, 4 p.

[16] J. Dressel and A. N. Jordan, "Contextual-Value Approach to the Generalized Measurement of Observables," Physical Review A, Vol. 85, No. 2, 2012, 32 p. doi:10.1103/PhysRevA.85.022123

[17] M. Ozawa, "Universal Uncertainty Principle, Simultaneous Measurability, and Weak Values," AIP Conference Proceedings, Brisbane, 19 July 2011, pp. 53-62.

[18] K. Svozil, “Quantum Logic," Springer-Verlag, Singapore City, 1998

[19] S. Maeda, "Lattice Theory and Quantum Logic," MakiShoten, Tokyo, 1980. 